\title{
High Resolution Magic Angle Proton Magnetic Resonance Spectroscopy (HRMAS) in Intact Sentinel Node Biopsy from Breast Cancer Patients: A New Diagnostic Tool!
}

Surender Kumar ${ }^{*}$, Shailendra Kumar ${ }^{1}$, Raja Roy ${ }^{2}$, Ankita Singh Rathore ${ }^{1}$, Madhu Mati Goel ${ }^{3}$, Gaurav Agarwal ${ }^{4}$ and Sandeep Kumar ${ }^{1}$

${ }^{1}$ Department of Surgery, King Georges Medical University, Lucknow, India

${ }^{2}$ Center of Biomedical Magnetic Resonance, Sanjay Gandhi Postgraduate Institute of Medical Sciences, Lucknow, India

${ }^{3}$ Department of Pathology, King Georges Medical University, Lucknow, India

${ }^{4}$ Department of Endocrine Surgery, Sanjay Gandhi Postgraduate Institute of Medical Sciences, Lucknow, India

\begin{abstract}
Introduction: The extent of axillary lymph node involvement is one of the most important prognostic markers in patients of breast cancer. However, axillary dissection is associated with significant morbidity. The intra-operative sentinel node biopsy (SNB) provides a basis for omitting the routine axillary clearance however; use of in-house frozen section histopathology is required in order to substitute later. We report the use of in vitro high resolution magic angle proton magnetic resonance spectroscopy (HRMAS) for assessing the axillary nodal status with increased sensitivity.
\end{abstract}

Methods: Freshly removed axillary lymph nodes $(n=17)$ obtained during sentinel node biopsy from 17 different patients were bisected. One segment of the bisected node was snap frozen and transported to HRMAS laboratory and was blindly subjected to $400 \mathrm{MHz}$ high resolution magic angle proton magnetic resonance spectroscopy. The other portion was sent for frozen section biopsy. The tissues specimens used for HRMAS analysis and remaining portion of bisected node were then formalin fixed, paraffin embedded and sent for histo-pathological examination in separate vials. The metabolic profiles of these nodes were correlated with the routine histo-pathogical findings.

Results: On histo-pathological examination, 7 nodes were found to be positive for metastasis were as 10 nodes were negative. The spectra of nodes $(n=7)$ found to be positive for malignant cells were exclusively dominated by signals from choline, choline containing compounds and lactate in the spectral region of $3.2 \mathrm{ppm}$ and $4.12 \mathrm{ppm}$ respectively. Overall the sensitivity and specificity of HRMAS in the present study was $100 \%$.

Conclusion: Metastatic and non-involved lymph nodes in breast cancer can be accurately distinguished based on its metabolic profile. The technique of high resolution magic angle proton magnetic resonance spectroscopy can be utilized in enhancing the sensitivity and specificity of sentinel node biopsy and may replace frozen section histopathology

Keywords: High resolution magic angle spectroscopy; Sentinel node; Breast cancer

\section{Introduction}

The extent of axillary lymph node involvement in breast cancer is a dominant indicator for systemic failure [1]. Therefore an adequate axillary lymph node dissection (ALND) along with mastectomy or breast conserving procedures is an established way to achieve a cure. However the procedure carries a significant morbidity in the form of sensory neuropathy, loss of shoulder mobility, shoulder pain and lymphedema of breast and arm [2].

Intra operative frozen section histopathology of the sentinel node (s) is an alternative to the standard axillary clearance however it has variable sensitivity of $60 \%$ to $95 \%[3,4]$. Various methods such as immuno- staining for cytokeratin have been included along with frozen section histopathology to increase the sensitivity of the sentinel node biopsy [5].

The diagnostic workups using patho-morphological changes to some extent can be substituted by molecular diagnostics techniques. Molecular techniques of proteonomics, genomics and metabonomics have emerged as possible alternative or adjudicative to histomorphological tests [6-8]. The term metabonomics is defined as "the quantitative measurement of the dynamic multiparametric metabolic response of living system to pathophysiologic stimuli or genetic modification". Magnetic resonance spectroscopy has emerged out as one of the main techniques of metabonomics and has been widely used to asses the health risk of particular drug/toxins. The in vitro and in vivo application of magnetic resonance spectroscopy for the diagnosis and therapeutic monitoring of various medical and surgical conditions like the hydatid diseases, leishmaniasis, diagnosis of malabsorption syndrome and liver graft dysfunction have been described in the past [9-12]. The conventional technique of magnetic resonance spectroscopy [MRS] required separation of analytes by preparing time consuming and labour intensive tissue extracts. Furthermore, the specimen gets consumed in the process and is unavailable for histopathological examination. The technique of high resolution proton magic angle spinning (HRMAS) spectroscopy however is yet another advancement to analyze the metabolic profile of an intact specimen and has been used successfully to differentiate malignant breast tissue from adjacent normal tissue on the basis of metabolic finger prints [13]. The quick

*Corresponding author: Surender Kumar, Department of General Surgery, King Georges Medical University, Lucknow, India- 226 003, Tel: +9190050112189, E-mail:dr_kukku@yahoo.co.in

Received July 17, 2014; Accepted January 03, 2015; Published January 09 2015

Citation: Kumar S, Kumar S, Roy R, Rathore AS, Goel MM. High Resolution Magic Angle Proton Magnetic Resonance Spectroscopy (HRMAS) in Intact Sentinel Node Biopsy from Breast Cancer Patients: A New Diagnostic Tool!. Journal of Surgery [Jurnalul de chirurgie] 2015; 11(2): 363-367 DOI: 10.7438/1584-9341-11-2-2

Copyright: (C) 2015 Kumar S, et al. This is an open-access article distributed under the terms of the Creative Commons Attribution License, which permits unrestricted use, distribution, and reproduction in any medium, provided the original author and source are credited. 
processing time is an added advantage that prompted us to evaluate the value of HRMAS spectroscopy vis- $a$ vis frozen section histopathology in sentinel node assessment from $\mathrm{T}_{1} / \mathrm{T}_{2}$ breast carcinoma patient in a pilot study.

\section{Method}

The study was carried out at the Department of General Surgery, CSM (King George's) Medical University, Lucknow and NMR Laboratory, Sophisticated Analytical Institute Facility, Central Drug Research Institute, Lucknow. The study was duly approved by the institutional ethical committee.

Sentinel node biopsy was performed in 17 patients by injecting blue dye (iso-sulphan blue 1\%) in the subareolar region in patients planned for local wide excision for $T_{1} T_{2}$ lesions with clinically negative axillae. None of the patients had received chemotherapy.

One largest blue node was taken for this study (Figure 1). The node was thoroughly washed in normal saline, bisected and one part was used for frozen section biopsy (Figure 2) and other was put into a cryogenic vial with a unique ID code. The vial was immediately snap frozen and carried in a liquid nitrogen container to the NMR laboratory facility $(1.5 \mathrm{kms})$. The node was thinly sliced using a sharp surgical blade. A large slice about 2 to $3 \mathrm{~mm}$ thick and weighing $35-40 \mathrm{mg}$ was put into $4 \mathrm{~mm}$ HRMAS rotor. The lymph node slice was assembled inside the rotor and 20 micro liter of $\mathrm{D} 2 \mathrm{O}$ containing tri methyl silyl tetra deuteron propionic acid (TSP) was added as standard. The HRMAS experiments were carried out on a Bruker Avance $400 \mathrm{MHz}$ FT NMR spectrometer equipped with $4 \mathrm{~mm}{ }^{1} \mathrm{H}$ and ${ }^{13} \mathrm{C}$ dual HRMAS with magic angle gradient at $4^{\circ} \mathrm{C}$. The samples were spun at $4.0 \mathrm{KHz}$ in order to keep rotation side bands out of the acquisition window.

One dimensional proton NMR spectra with water pre-saturation were acquired using NOESY pulse sequence with a mixing time of $\tau \mathrm{m}$ 100 milli seconds. Total relaxation delay of $3.99 \mathrm{sec}$ was used using $8250.8 \mathrm{~Hz}$ spectral width, 128 transients with a total recording time of 9.44 minutes. The one dimensional CPMG pulse sequence with water pre-saturation using an echo time of 200 milli seconds was used in order to filter off short T2 lipid component. Each experiment took about 20 minutes and the spectra were available for study on the dedicated computer screen. Assignments of HRMAS spectra were done as per published data [14]. The HRMAS spectra were read by the NMR expert (RR) who was not aware of the histopathological diagnosis of the sample. He was asked to deliver and sign a report in the shortest possible time to mimic the per-operative frozen section histopathology scenario. It may be noteworthy that routine frozen section facility was not available in our institution at the time of study. Completion axillary dissection was carried out in all 17 subjects.

The tissue used for the study was retrieved from the HRMAS rotor it was then formalin fixed and was taken for histopathological examination by standard $\mathrm{H} \& \mathrm{E}$ staining. The histopathology results were generally available a week later. The remaining tissue slices were also formalin fixed and sent separately for histipathological examination.

\section{Results}

Seventeen lymph node specimens from 17 subjects undergoing sentinel node biopsy from $\mathrm{T}_{1} / \mathrm{T}_{2}$ clinically $\mathrm{N} 0$ breast carcinoma patients were taken for the study. Each patient in this group yielded only one worthwhile lymph node for the study. The mean size of the nodes used for HRMAS study was $0.68 \mathrm{cms}$. Of all the differences in the cellular metabolism detected the high peaks of the metabolites viz; choline $(\mathrm{Ch})$ and choline containing compounds eg; phosphocholine and phosphatidyl choline in the region of $3.2 \mathrm{ppm}$ in $7 / 17$ slices were most prominently seen (Figure 3 ). The presence of lactate at 4.12 ppm suggesting a raised anaerobic metabolism was also seen in all these 7 nodes. Furthermore, high concentration of amino acids was also observed in all these nodes. A portion of stack plot of the CPMG spectra depicting the presence of amino acid; glycine is shown in Figure 4. In 10/17 slices that were examined using the same HRMAS experiment in a clear contrast did not show the above metabolites. The NMR laboratory results could be interpreted almost instantaneously and one was quick to point out the above described differences and overtly 2 types of spectra. The HRMAS study data print outs in all the specimens examined from time to time were available within 30 minutes of receiving the tissue from the operating room.

The tissue slices retrieved from the NMR rotor were subjected to histopathology using $\mathrm{H} \& \mathrm{E}$ stain. There was clear and unequivocal evidence of malignancy in $7 / 17$ nodes and none in $10 / 17$ nodes. The histopathology of the remaining lymph node tissue slices also corroborated with the core slice examined. Upon decoding and correlating the histomorphological data with HRMAS findings it was obvious that overall the technique of HRMAS was $100 \%$ sensitive and $100 \%$ specific in these experiments (Table 1).

\section{Discussion}

This study reports a rather new efficient method of detecting tumor in sentinel node biopsy specimen from early stage breast cancer patients. The in-vitro use of High Resolution Magic Angle Spectroscopy on intact lymph node slice within 30 minutes from the operating room may be an important alternative to frozen section histopathological examination. Molecular diagnostic markers are an emerging field in cancer diagnosis and prognostic predictions. The in vitro technique of intact tissue metabonomics using HRMAS studies was able to differentiate cancer from non-cancer in this small sample of lymph nodes.

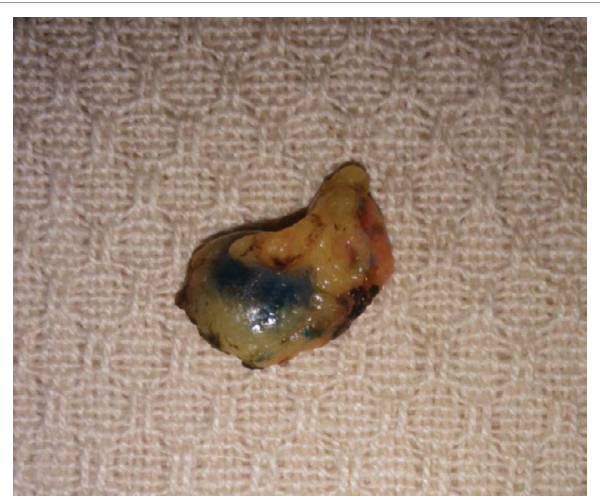

Figure 1: Extracted Blue Node

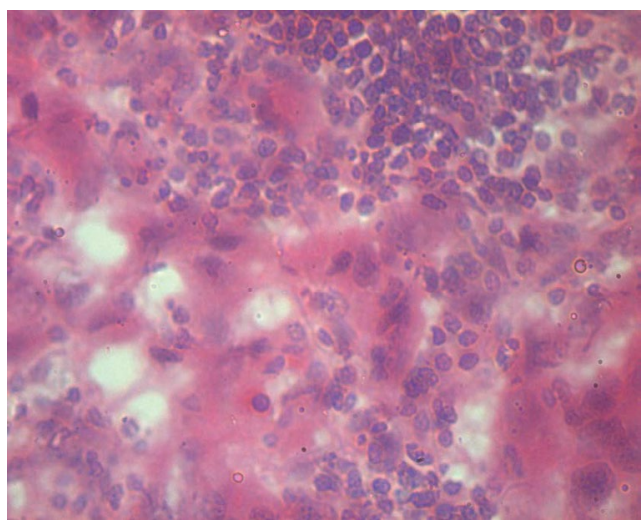

Figure 2: Frozen Section Biopsy of Involved Node. 


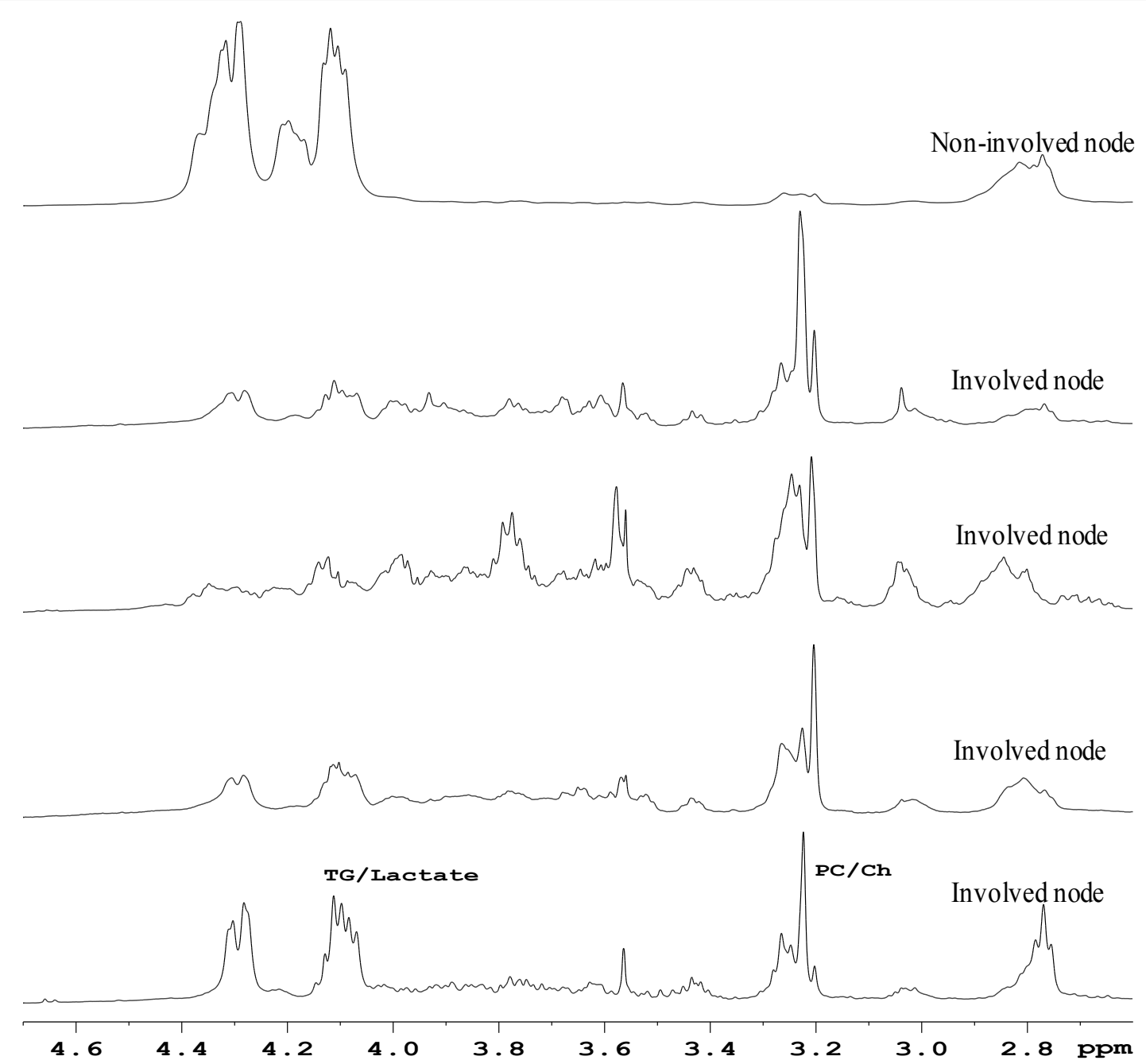

Figure 3: A Portion of 1D NOESY Stack Plot Spectra of Involved and Non-involved Lymph Nodes (PC- Phospocholine, Ch- Choline, TG- Tri Glycride)

Choline and choline containing compounds are present in more than one tumor type but these metabolites are characteristically absent in normal and benign tissues [15,16]. Identification of cancerous secondary in an axillary sentinel lymph node as opposed to a non affected node indeed demonstrated a practical use of this knowledge. Thus, of all the alterations in the metabolic profile detected by the HRMAS spectra, the region of $3.2 \mathrm{ppm}$ demonstrated the most useful metabolite choline, choline containing compound (phosphocholine) and lactate at $4.12 \mathrm{ppm}$. Choline and its derivatives are one of the important building blocks of cell physiology and represent accelerated cell proliferation in the presence of malignancy. Similarly choline has been detected in breast cancer tissue extract and ex-vivo MRS studies of breast cancer $[17,18]$. The choline and choline containing compounds signal were reported to be less prominent in patients of breast cancer treated with neo adjuvant chemotherapy [19].

Choline and choline containing compounds were also reported from lactating breast tissue $[13,20]$. Whether axillary lymph nodes in lactating women also show high choline peaks is unknown. The state of lactation is associated with increased choline metabolism because of the need to nourish the newborn with large amounts of choline (supplied in the milk predominantly as phosphatidylcholine, phosphocholine, glycerophosphocholine, and free choline). This may limit the specificity of choline and choline containing compounds as discriminating metabolite in lactating breast carcinoma, a rarity, however.

The second important discriminating metabolite in this study was the lactate peaks at $4.12 \mathrm{ppm}$. In malignant cells the anaerobic metabolism of glucose is the major metabolic process and is thought to be responsible for the raised level of lactate [21].

The above described metabolites were indeed well known to occur in tissue extracts of cancers of several organs notably, breast, oral squamous cell carcinoma, urinary bladder and proste cancer, brain tumours etc. Most of the earlier studies were done on cellular extracts of tumour in the early nineties. The metabonomics of intact tissue slices referred to as ex vivo in vitro tissue metabonomics is a rather recent development. The HRMAS technique was used to diagnose primary breast tumour and showed a high correlation with histopathology. The breast carcinoma tissue and juxta cancer tissue free from cancer were distinguished on the basis of these spectra [22]. Choline, lactate and other metabolites were significantly elevated in the malignant tissue. This study reported from China claimed it as a new technique for the diagnosis of human breast cancer in addition to histopathology. However there is a limitation for the use of lactate as a sole marker of malignancy in the detached specimens as it may be produced anaerobically and give a false positive result. Combined presence of choline and lactate along with other metabolites like creatinine, betaglucose, GPC, glycine, myo-inositol and taurine were suggested as a sum total marker of malignancy with greater degree of confidence or diagnostic sensitivity in tissue samples.

The clinical radiologists can also do metabolites estimation from their standard in vivo magnetic resonance imaging (MRI) machine by simply using software. Such an in-vivo HRMAS in which the patient 

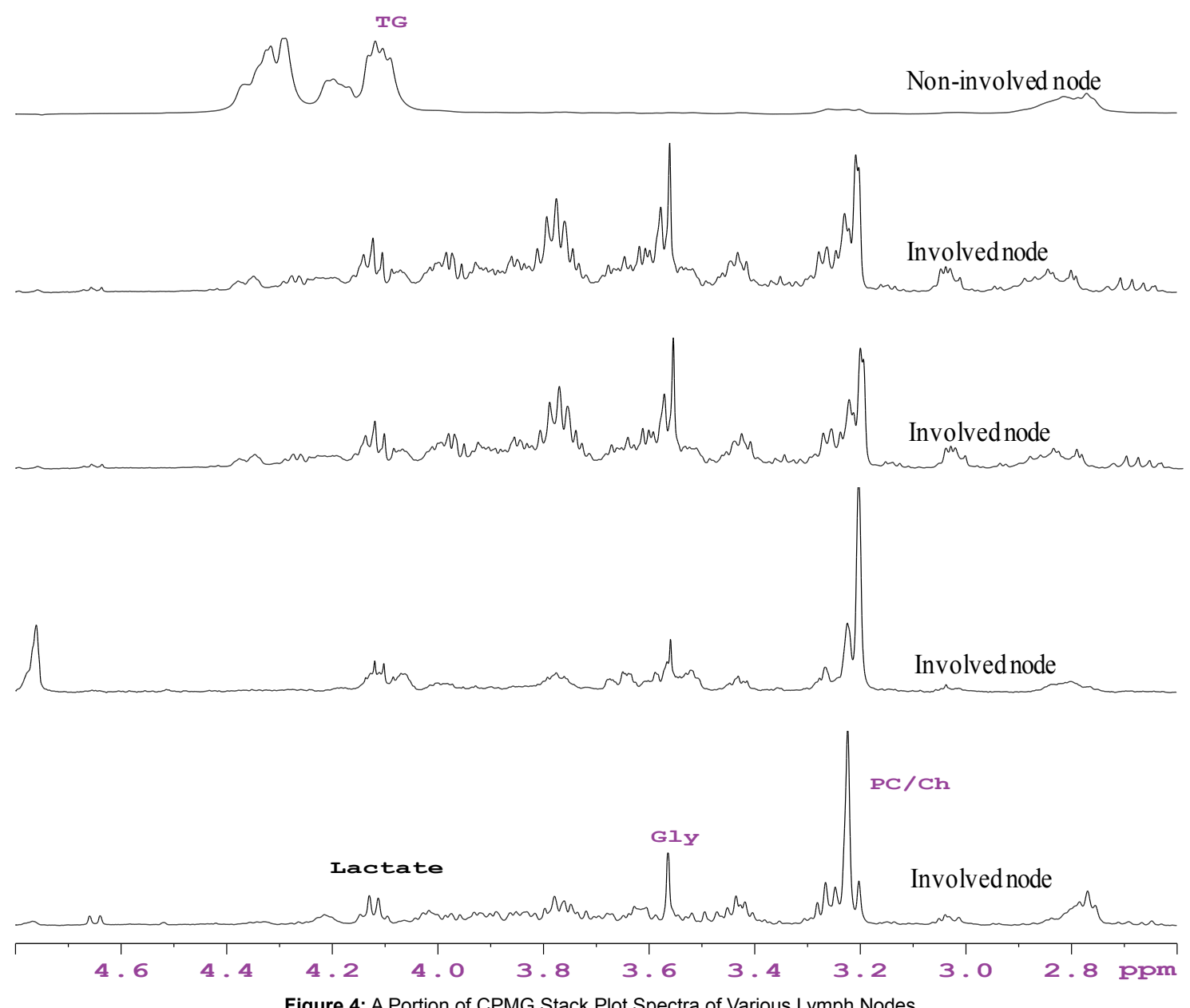

Table 1: Test Results of HRMAS Compared with Histopathological Examination

\begin{tabular}{|c|c|c|c|}
\hline & HPE +ve & HPE -ve & Total \\
\hline HR MAS +ve & 7 & 0 & 7 \\
\hline HR MAS -ve & 0 & 10 & 10 \\
\hline Total & 7 & 10 & \\
\hline
\end{tabular}

Sensitivity: $100 \%$

Specificity: $100 \%$

Positive predictive value: $100 \%$

Negative predictive value: $100 \%$

herself goes into the MRI machine and the metabolic profile of the tumor is recorded with the help of special software has been evaluated in axillary nodes in breast cancer in 2 studies [23,24]. Firstly, in 35 nodes using the basis of choline signals alone the authors identified metastasis with sensitivity, specificity and accuracy of $82 \%, 100 \%$ and $90 \%$ [23]. In the second study, using the similar technique in 20 nodes the sensitivity, specificity and accuracy of $80 \%, 91 \%$, and $88 \%$ were described. It was further noted in these studies conducted longitudinally on these patients that neo-adjuvant chemotherapy lowered the concentration of various metabolites e.g. choline, phosphocholine, etc. [24]. As these patients were undergoing chemotherapy and longitudinal assessment by MRI these describe a different subset of patients in whom rather large axillary lymph nodes were present as opposed to the non palpable nodes in the axillae of the patients that have been included in the present study. The ability of MRI to detect a sentinel node in clinically N0 axillae of early breast carcinoma has not been evaluated. The MRI along with in vivo MRS in clinically N0 axillae can theoretically be a tool for pre operative detection of malignancy in a sentinel node.
The molecular diagnosis of malignant change as opposed to histomorphological diagnosis is an emerging field of medical research. Metabnomics by MRS equipments are generally expensive commodity largely available in big public sector hospitals and corporate pharmaceuticals industries so far. There is relative paucity of trained man power in the field of MRS. Pharmacological companies and chemical industries routinely use MRS to test the purity of their products. Clinician's interest however, in this field has been tardy. An increasing interest and availability of the MRS equipment is being witnessed world over. The MRS or in vivo MRS and MRI as common facility can become cost effective I future with increasing usage. The invitro per-operative expeditious assessment HRMAS of an intact axillary lymph node slice or other tissues as a central facility for a number of hospitals in the vicinity of the HMRS facility can be promoted as a cost effective technology. Several other applications of in- vitro HRMAS studies include detection of micro-metastases, ability to detect residuals in the tumor bed and tumor margins, metabolites in tumor aspirate, exfoliated cells metabonomics, brush cytology specimens and, fluids like bronchial lavage, asicitic tap and nipple discharge. The initial fixed cost of the equipment though high is also likely to go down with widespread use in the future. Though at present the discriminating metabolites for a particular type of carcinoma or sarcoma are not available, the study designs like the present study offer a useful application of this technology within the present level of knowledge. This study was conducted to mimic the scenario of sentinel node assessment in the operating theatre. To this effect HMR spectroscopy emerged as an efficient and reliable method for the evaluation of the sentinel node as compared with routine histopathology. A larger sample size on a even higher frequency MRS $(800 \mathrm{MHz})$ equipment may further enhance these results. 


\section{Acknowledgement}

1. Financial assistance from ICMR New Delhi, India via grant no. 5 / 13/96/ 2003 - NCD III , IRIS No : $2003-04710$

2. We are grateful to the NMR division of SAIF, CDRI, Lucknow for providing the NMR facility and thankful to MR HM Guniyal for recording the HR-MAS NMR spectra of the lymph node specimens.

\section{References}

1. Henderson IC, Patek AJ (1998) The relationship between prognostic and predictive factors in the management of breast cancer. Breast Cancer Res Treat 52: 261-288.

2. Duff M, Hill AD, McGreal G, Walsh S, McDermott EW, et al. (2001) Prospective evaluation of the morbidity of axillary clearance for breast cancer. $\mathrm{Br} \mathrm{J}$ Surg 88: 114-117.

3. Tanis PJ, Boom RP, Koops HS, Faneyte IF, Peterse JL, et al. (2001) Frozen section investigation of the sentinel node in malignant melanoma and breast cancer. Ann Surg Oncol 8: 222-226

4. Layfield DM, Agrawal A, Roche H, Cutress RI (2011) Intraoperative assessmen of sentinel lymph nodes in breast cancer. Br J Surg 98: 4-17.

5. Zurrida S, Mazzarol G, Galimberti V, Renne G, Bassi F, et al. (2001) The problem of the accuracy of intraoperative examination of axillary sentinel nodes in breast cancer. Ann Surg Oncol 8: 817-820.

6. Bernini A, Spencer M, Frizelle S, Madoff RD, Willmott LD et al. (2000) Evidence for colorectal cancer micrometastasis using reverse transcriptase polymerase chain reaction analysis of MUC2 in lymph nodes. Cnacer Detect Prev 24: 72-79.

7. Liefers GJ, Tollenaar RA, Cleton-Jansen AM (1999) Molecular detection of minimal residual disease in colorectal and breast cancer. Histopathology 34 : $385-390$

8. Cohn KH, Ornstein DL, Wang F, LaPaix FD, Phipps K, et al. (1997) The significance of allelic deletions and aneuploidy in colorectal carcinoma. Results of a 5-year follow-up study. Cancer 79: 233-244.

9. Garg M, Gupta RK, Prasad KN, Sikora SS, Pal L, et al. (2002) Fertility assessment of hydatid cyst by proton MR spectroscopy. J Surg Res 106: 196-201.

10. Gupta N, Goyal N, Singha UK, Bhakuni V, Roy R, et al. (1999) Characterization of intracellular metabolites of axenic amastigotes of Leishmania donovani by 1H NMR spectroscopy. Acta Trop 73: 121-133.

11. Bala L, Nagana Gowda GA, Ghoshal UC, Misra A, Bhandari M, et al. (2004) 1H NMR spectroscopic method for diagnosis of malabsorption syndrome: a pilot study. NMR Biomed 17: 69-75.
12. Singh HK, Yachha SK, Saxena R, Gupta A, Nagana Gowda GA, et al. (2003) A new dimension of $1 \mathrm{H}-\mathrm{NMR}$ spectroscopy in assessment of liver graft dysfunction. NMR Biomed 16: 185-188.

13. Kvistad KA, Bakken IJ, Gribbestad IS, Ehrnholm B, Lundgren S, et al. (1999) Characterization of neoplastic and normal human breast tissues with in vivo (1) H MR spectroscopy. J Magn Reson Imaging 10: 159-164.

14. Sitter B, Sonnewald U, Spraul M, Fjösne HE, Gribbestad IS (2002) Highresolution magic angle spinning MRS of breast cancer tissue. NMR Biomed 15: 327-337.

15. Stanwell P, Gluch L, Clark D, Tomanek B, Baker L, et al. (2005) Specificity of choline metabolites for in vivo diagnosis of breast cancer using $1 \mathrm{H}$ MRS at 1.5 T Eur Radiol 15: 1037-1043.

16. Rutter A, Hugenholtz H, Saunders JK, Smith IC (1995) Classification of brain tumors by ex vivo 1H NMR spectroscopy. J Neurochem 64: 1655-1661.

17. Gribbestad IS, Fjösne HE, Haugen OA, Nilsen G, Krane J, et al. (1993) In vitro proton NMR spectroscopy of extracts from human breast tumours and noninvolved breast tissue. Anticancer Res 13: 1973-1980.

18. Kumar M, Jagannathan NR, Seenu V, Dwivedi SN, Julka PK, et al. (2006) Monitoring the therapeutic response of locally advanced breast cancer patients: sequential in vivo proton MR spectroscopy study. J Magn Reson Imaging 24 325-332.

19. Jagannathan NR, Kumar M, Seenu V, Coshic O, Dwivedi SN, et al. (2001) Evaluation of total choline from in-vivo volume localized proton MR spectroscopy and its response to neoadjuvant chemotherapy in locally advanced breast cancer. Br J Cancer 84: 1016-1022.

20. Stanwell P, Gluch L, Clark D, Tomanek B, Baker L, et al. (2005) Specificity of choline metabolites for in vivo diagnosis of breast cancer using $1 \mathrm{H}$ MRS at 1.5 T. Eur Radiol 15: 1037-1043.

21. Gribbestad IS, Fjösne HE, Haugen OA, Nilsen G, Krane J, et al. (1993) In vitro proton NMR spectroscopy of extracts from human breast tumours and noninvolved breast tissue. Anticancer Res 13: 1973-1980.

22. Cheng LL, Chang IW, Smith BL, Gonzalez RG (1998) Evaluating human breast ductal carcinoma with High-Resolution Magic Angle Spinning Proton Magnetic Resonance Spectroscopy. J Magn Reson 135: 194-202

23. Yeung DK, Yang WT, Tse GM (2002) Breast cancer: in vivo proton MR spectroscopy in the characterization of histopathologic subtypes and preliminary observations in axillary node metastases. Radiology 225: 190-197.

24. Seenu V, Pavan Kumar MN, Sharma U, Gupta SD, Mehta SN, et al. (2005) Potential of magnetic resonance spectroscopy to detect metastasis in axillary lymph nodes in breast cancer. Magn Reson Imaging 23: 1005-1010.
Submit your next manuscript and get advantages of OMICS Group submissions

Unique features:

User friendly/feasible website-translation of your paper to 50 world's leading languages

Audio Version of published paper

Digital articles to share and explore

Special features:

400 Open Access Journals

30,000 editorial team

21 days rapid review process

Quality and quick editorial, review and publication processing

Indexing at PubMed (partial), Scopus, EBSCO, Index Copernicus and Google Scholar ete

Sharing Option: Social Networking Enabled

Authors, Reviewers and Editors rewarded with online Scientific Credits

Better discount for your subsequent articles

Submit your manuscript at: http://www.omicsonline.org/submission 